\title{
Low expression of circulating microRNA-328 is associated with poor prognosis in patients with acute myeloid leukemia
}

\author{
Li Liu*, Ren'an Chen, Yangping Zhang, Wen Fan, Fang Xiao and Xueqian Yan
}

\begin{abstract}
Background: Dysregulation of circulating miR-328 has been identified in several tumors and is associated with prognosis of patients. However, the expression pattern of miR-328 and the impact on prognosis has not yet been studied in acute myeloid leukemia (AML). The purpose of this study is to investigate the expression status of miR-328 and its clinical significance in AML patients.

Methods: RNA was extracted from plasma of 176 patients with newly diagnosed AML and 70 healthy volunteers. The miR-328 expression was examined by Realtime quantitative PCR. The association of circulating miR-328 expression with clinicopathological factors and prognosis of AML patients was statistically analyzed.

Results: The expression of miR-328 was significantly downregulated in AML patients (median value 22.99, range: 3.63-242.0) compared with those of healthy controls (median value 89.17, range: $12.05-397.7 ; P<0.001$ ), and miR-328 expression was markedly increased in patients after treatment than before ( $23.40 \pm 1.76$ vs. $46.61 \pm 3.83$, $P<0.001)$. Moreover, low levels of miR-328 were associated with a higher white blood cell count and BM blast count $(P=0.026$ and $P=0.003$, respectively), and lower hemoglobin and platelet count $(P=0.004$ and $P=0.022$, respectively). Patients with low miR-328 expression had a relatively poor overall survival $(P=0.022)$ and shorter relapse-free survival $(P=0.008)$ than those with high miR-328 expression. In addition, low miR-328 expression was an independent prognostic factors for both OS $(P=0.017)$ and RFS $(P=0.023)$.
\end{abstract}

Conclusions: Circulating miR-328 downregulation is a common event and is associated with poor clinical outcome in AML patients.

Keywords: Circulating, miR-328, Acute myeloid leukemia, Prognosis

\section{Background}

Acute myeloid leukemia (AML), the most common type of acute leukemia in adults, is a clonal disorder caused an accumulation and differentiation arrest of myeloid blasts in the bone marrow and blood. The pathologic mechanism of AML can be largely explained by cytogenetic aberrations, acquired mutations and dysregulated gene expression $[1,2]$. Based on cytogenetic information, AML patients are classified into three risk-based categories: favorable, intermediate, and poor, with a 5-year overall survival (OS) rate of $55 \%, 24 \%$ $42 \%$, and $11 \%$, respectively [3]. Treatment of AML has

\footnotetext{
* Correspondence: lliu6699@126.com

Department of Haematology, Tangdu Hospital, Fourth Military Medical University, No.1, Xinsi Road, Xi'an, Shaanxi 710038, People's Republic of China
}

dramatically improved over the past several decades, with improvements in risk assessment, post-remission chemotherapy and hematopoietic stem-cell transplantation. However, the cause of AML is not yet fully understood. Therefore, early and accurate diagnosis of AML is essential for optimal treatment outcome and may deeply improve the prognosis of patients with AML.

MicroRNAs (miRNAs) are a class of non-coding small RNAs of $\sim 22$ nucleotides that regulate expression of target genes at the post-transcriptional level [4]. MicroRNAs function by directly binding to their potential target site in the 3'untranslated region (3'UTRs) of specific target mRNAs, resulting in the repression of mRNA translation or the degradation of target mRNAs [5]. Since the discovery of the first miRNAs, these small genes have 
added a new layer of complexity to the regulation of normal and pathological cell functions. Recent studies have indicated a key role of miRNAs in biological processes including cell proliferation, differentiation, apoptosis, as well as cancers and cardiovascular diseases [5, 6]. Currently, aberrant expression of miRNAs appears to be a common characteristic of hematological malignancies, including leukemias $[7,8]$. Dysregulation of single miRNAs such as miR-212 [9], miR-124-1 [10], miR-181 [11] and let-7a-3 [12] has been found to be associated with the outcome of AML patients.

Recently, it has been reported that miRNAs are present in serum or plasma in a stable and reproducible fashion, and the unique expression patterns of serum or plasma miRNAs can be used as a new class of effective biomarkers for various diseases [13-15]. MiR-328, known as a tumor suppressor, is involved in the cancer development and progression $[16,17]$. MiR-328 was reported down-regulated in chronic myelogenous leukemia blasts and glioblastoma tissues. However, a previous report found that peripheral blood miR-328 expression was up-regulated in non-small cell lung cancer (NSCLC) patients [18]. Wang et al. found that plasma miR-328 concentrations were significantly elevated in acute myocardial infarction (AMI) patients compared to those control subjects [19].

However, to the best of our knowledge, no previous reports exist concerning the expression status of circulating miR-328, the prognostic value and the role of this miRNA in AML. Thus, the aim of the present study was to investigate the correlation of circulating miR-328 with clinicopathological features as well as the prognosis of the patients with AML. Our findings may provide the better understanding on the roles and its clinic implications of circulating miR-328 in the development and progression of AML.

\section{Methods}

\section{Patients and follow-up}

From February 2010 to September 2014, 176 newly diagnosed de novo AML patients from the Department of Hematology at Tangdu Hospital of Fourth Military Medical University were enrolled in this study; there were 86 males and 90 females, with a medium age of 39.7 (range 16.2-67.6) years. 70 unrelated healthy adult donors were collected as controls; all the control subjects were matched with patient population in terms of age and sex. None of these controls had previously been diagnosed with any type of malignancy or other benign disease. AML patients were diagnosed according to standard diagnostic methods including cytomorphological, cytochemical, immunological and cytogenetic evaluation. The diagnosis and classification of AML patients were based on the French-American-British (FAB) and World Health Organization (WHO) criteria combined to immunophenotyping and cytogenetic analysis [20-23]. 124 patients received standard induction chemotherapy consisted of 1 or 2 courses of daunorubicin (45 $\mathrm{mg} / \mathrm{m}^{2}$ daily for 3 days) combined with cytarabine $\left(100 \mathrm{mg} / \mathrm{m}^{2}\right)$ by a 7 -day continuous intravenous infusion. AML complete remission (CR) was defined as a normocellular BM containing less than $5 \%$ blasts and showing evidence of normal maturation of other marrow elements; a neutrophil count of $1 \times 10^{9} / \mathrm{L}$ and a platelet count of $100 \times 10^{9} /$ L. 76 patients achieved CR, and then given high- or medium dose cytarabine-based chemotherapy for consolidation according to their physical condition. Patients were followed up for a median 26 months (range 5-51 months); Patients without death or relapse by the time of last follow-up were censored on that date. Overall survival (OS) was defined as the time from the diagnosis of AML to any cause of death. Relapse-free survival (RFS) was defined as the time between the achievement of complete remission and the time of the hematological relapse or death. This study was approved by the Ethics Committee Board of Tangdu Hospital of Fourth Military Medical University. Informed consent was obtained from each participant according to the committee's regulations. Details of clinical characteristics of the patients are provided in Table 1.

\section{Plasma collection and RNA extraction}

Blood samples were collected in EDTA- $K_{2}$ tubes and processed within $1 \mathrm{~h}$ of collection. Cell and nucleic acids free plasma was isolated from all blood samples using a 2-step centrifugation protocol $(3000 \mathrm{~g}$ for $10 \mathrm{~min}$ and $12000 \mathrm{~g}$ for $5 \mathrm{~min}$, all at $4{ }^{\circ} \mathrm{C}$ ). The supernatant was transferred to RNase/DNase free tubes and stored at $-80{ }^{\circ} \mathrm{C}$. The plasma was first spiked with miScript miRNA mimic SV40 (Qiagen, Hilden, Germany, $2 \mu \mathrm{M}$, $1 \mu \mathrm{l}$ per $100 \mu \mathrm{l}$ plasma). Total RNA was isolated from the plasma using TRI reagent BD (MRC, USA) according to the manufacturer's instructions and dissolved in $20 \mu \mathrm{l}$ of RNase-free water. RNA sample concentration was quantified by NanoDrop ND-2000 (Thermo Fisher Scientific, USA). Quality of RNA was generally checked by the ratios of A260/A280 and A260/A230 and RNA integrity was assessed by electrophoresis through denaturing agarose gels.

\section{qRT-PCR analysis of plasma miR-328}

Total RNA $(1 \mu \mathrm{g})$ from each sample were converted into cDNA using PrimeScript RT reagent kit with gDNA Eraser (TaKaRa, Japan) and miRNA-specific stem-loop RT primer or SV-40 primers (Applied Biosystems, USA). Briefly, the reverse transcription reaction was performed in $20 \mu \mathrm{L}$ mixture containing $10 \mu \mathrm{L}$ of genomic DNA elimination reaction solution, 
Table 1 Clinicopathological variables of 176 patients with newly diagnosed AML and expression of miR-328

\begin{tabular}{|c|c|c|c|c|}
\hline \multirow[t]{2}{*}{ Clinicopathological variables } & \multirow[t]{2}{*}{ Cases $(176, \mathrm{n} / \%)$} & \multicolumn{2}{|c|}{ miR-328 expression } & \multirow[t]{2}{*}{$P$ value } \\
\hline & & low (125) & high (51) & \\
\hline \multicolumn{5}{|l|}{ Age (years) } \\
\hline$\leq 60$ & $126(71.6)$ & $90(72 \%)$ & $36(70.6 \%)$ & \multirow[t]{2}{*}{0.997} \\
\hline$>60$ & $50(28.4)$ & $35(28 \%)$ & $15(29.4 \%)$ & \\
\hline \multicolumn{5}{|l|}{ Gender } \\
\hline Male & $86(48.9)$ & $60(48 \%)$ & $26(51.0 \%)$ & \multirow[t]{2}{*}{0.847} \\
\hline Female & $90(51.1)$ & $65(52 \%)$ & $25(49.0 \%)$ & \\
\hline \multicolumn{5}{|l|}{ WBC $\left(\times 10^{9} / \mathrm{L}\right)$} \\
\hline$<10$ & $73(41.5)$ & $53(42.4 \%)$ & $30(58.9 \%)$ & \multirow[t]{2}{*}{0.026} \\
\hline$\geq 10$ & $103(58.5)$ & $82(57.6 \%)$ & $21(41.1 \%)$ & \\
\hline \multicolumn{5}{|l|}{$\mathrm{HGB}(\mathrm{g} / \mathrm{L})$} \\
\hline$<80$ & $110(62.5)$ & $87(69.6 \%)$ & $23(45.1 \%)$ & \multirow[t]{2}{*}{0.004} \\
\hline$\geq 80$ & $66(37.5)$ & $38(30.4 \%)$ & $28(54.9 \%)$ & \\
\hline \multicolumn{5}{|l|}{$\operatorname{PLT}\left(\times 10^{9} / \mathrm{L}\right)$} \\
\hline$<50$ & $91(51.7)$ & $72(57.6 \%)$ & $19(34.2 \%)$ & \multirow[t]{2}{*}{0.022} \\
\hline$\geq 50$ & $85(48.3)$ & $53(42.4 \%)$ & $32(62.8 \%)$ & \\
\hline \multicolumn{5}{|l|}{ Blast in BM } \\
\hline$<50 \%$ & $79(44.9)$ & $45(36 \%)$ & $34(66.7 \%)$ & \multirow[t]{2}{*}{0.003} \\
\hline$\geq 50 \%$ & $97(55.1)$ & $80(64 \%)$ & $17(33.3 \%)$ & \\
\hline \multicolumn{5}{|l|}{ FAB subtype } \\
\hline M1 & $35(19.9)$ & $25(20 \%)$ & $10(19.6 \%)$ & \multirow[t]{5}{*}{0.909} \\
\hline M2 & $44(25)$ & $32(22.6 \%)$ & $12(23.5 \%)$ & \\
\hline M3 & $11(6.2)$ & $7(5.6 \%)$ & $4(7.8 \%)$ & \\
\hline M4 & $26(14.8)$ & $20(16 \%)$ & $6(11.8 \%)$ & \\
\hline M5 & $60(34.1)$ & $41(32.8 \%)$ & $19(37.3 \%)$ & \\
\hline \multicolumn{5}{|l|}{ WHO Classification } \\
\hline AML with $\mathrm{t}(8 ; 21)$ & 19 (10.8) & $15(12 \%)$ & $4(7.8 \%)$ & \multirow[t]{6}{*}{0.074} \\
\hline APL with $t(15 ; 17)$ & $24(13.6)$ & $12(9.6 \%)$ & $12(23.5 \%)$ & \\
\hline AML without maturation & $15(8.5)$ & $8(6.4 \%)$ & $7(13.7 \%)$ & \\
\hline AML with maturation & $39(22.2)$ & $30(24 \%)$ & $9(17.6 \%)$ & \\
\hline Acute myelomonocytic leukemia & $26(14.8)$ & $21(16.8 \%)$ & $5(9.8 \%)$ & \\
\hline Acute monoblastic and monocytic leukemia & $53(30.1)$ & 39 (31.2 \%) & $14(23.6 \%)$ & \\
\hline \multicolumn{5}{|l|}{ Karyotype classification } \\
\hline Favorable & $72(40.9)$ & $56(44.8 \%)$ & $16(31.4 \%)$ & \multirow[t]{3}{*}{0.570} \\
\hline Intermediate & $83(47.2)$ & $55(44 \%)$ & $28(51.9 \%)$ & \\
\hline Unfavorable & $21(11.9)$ & $14(11.2 \%)$ & 7 (13.7 \%) & \\
\hline \multicolumn{5}{|l|}{ Complete Remission } \\
\hline Yes & $76(43.2)$ & 55 (44 \%) & $21(41.1 \%)$ & \multirow[t]{2}{*}{0.861} \\
\hline No & $100(56.8)$ & 70 (56 \%) & $30(58.9 \%)$ & \\
\hline
\end{tabular}

Note: WBC white blood cell, HGB hemoglobin, PLT platelet, FAB French-American-British, WHO World Health Organization

$4 \mu \mathrm{L} 5 \times$ PrimeScript Buffer, $1 \mu \mathrm{L}$ PrimeScript $\mathrm{RT}$ Enzyme Mix, $1 \mu \mathrm{L}$ stem-loop RT primer or SV-40 primers, and $2 \mu \mathrm{L}$ RNase Free water. For synthesis of $\mathrm{cDNA}$, the reaction mixture was incubated at $42^{\circ} \mathrm{C}$ for
$15 \mathrm{~min}, 85^{\circ} \mathrm{C}$ for $5 \mathrm{~s}$, and then held at $4{ }^{\circ} \mathrm{C}$. Quantitative reverse transcriptase polymerase chain reaction (qRT-PCR) was performed on ABI 7500 fast real-time PCR system (Applied Biosystems, USA) using SYBR Premix Ex Taq 
TM II (TaKaRa, Japan) according to the manufacturer's instructions. PCR program conditions were $95{ }^{\circ} \mathrm{C}$ for $30 \mathrm{~s}$, followed by 40 cycles of $95^{\circ} \mathrm{C}$ for $3 \mathrm{~s}$ and $60^{\circ} \mathrm{C}$ for $30 \mathrm{~s}$. A melting program was performed after each reaction to validate the specificity of the expected PCR product. The Ct values greater than 36 were considered as not expressed. Resultant miRNA levels were normalized using spiked-in SV40. The relative expression level of miR-328 was calculated by the equation of $2^{-\Delta C t}$ $\left(\Delta C_{t}=C_{t}\right.$ miR-328 $-C_{t}$ spiked-in SV40 $)$ [24]. The fold changes in miR-328 were calculated using the $2^{-\Delta \Delta \mathrm{Ct}}$ method [25]. Each sample was analyzed in triplicate and the mean expression level was calculated.

\section{Statistical analysis}

Statistical analysis was performed with SPSS 16.0 for Windows (SPSS, Chicago, IL). Continuous data are presented as mean \pm SD or median with interquartile range. Categorical variables are presented as counts and percentage. The Mann-Whitney U-test was used to evaluate the significant difference of expression of miR-328 between the AML patients and healthy controls. The paired $t$ test was used to evaluate the difference expression of miR-328 before and after chemotherapy. Chi-square analysis or Fisher exact test was performed to evaluate the difference of categorical variables. Univariate logistic regression analyses for the association with the risk of survival and relapse to AML were tested first for miR-328 expression, age, gender and other clinical characteristics, and those factors were included into a second multivariate logistic analysis. Survival curves were plotted using the Kaplan-Meier method, and differences were tested using the log-rank test. Differences were considered to be statistical significant when P value was less than 0.05 .

\section{Results}

\section{MiR-328 was downregulated in AML patients}

The miR-328 expression levels were detected in plasma samples from patients with AML and healthy controls by qRT-PCR. As shown in Fig. 1a, plasma concentration of miR-328 was markedly downregulated in AML patients (median expression value 22.99, range: 3.63-242.0) relative to those in healthy controls (median expression value 89.17, range: $12.05-397.7 ; P<0.001)$. In addition, 76 patients who achieved CR were monitored for miR-328 during the course of treatment. The mean fold change of miR-328 in these AML patients was markedly increased when CR was achieved after chemotherapy (mean expression value $23.40 \pm 1.76$ vs. $46.61 \pm 3.83, P<0.001$ ).

\section{Correlations between the levels of $\mathrm{miR}-328$ and the clinicopathological factors in AML patients}

To identify the clinical relevance of miR-328 expression in AML patients, correlations between miR-328 expression and clinicopathological parameters were made. AML patients expressing miR-328 at levels less than the mean expression level (33.1) were assigned to the low expression group (mean expression value 20.87, $\mathrm{n}=125$ ),
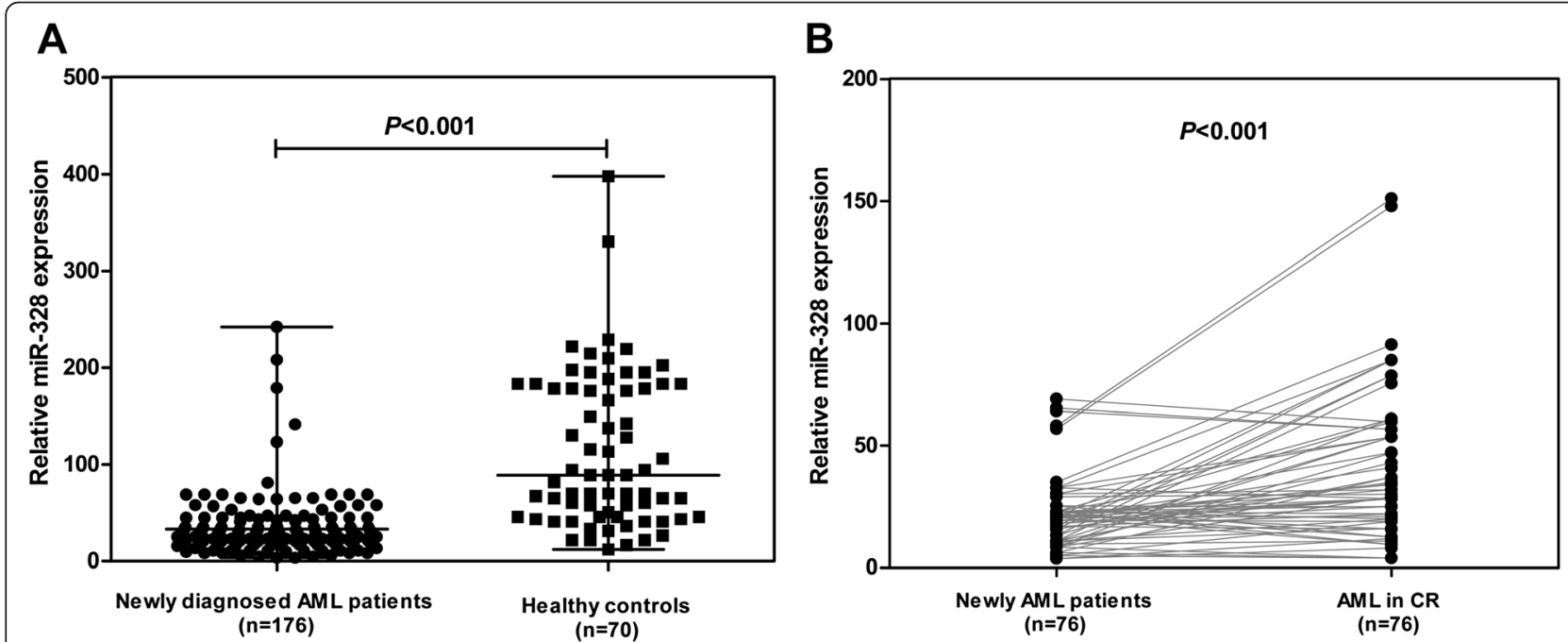

Fig. 1 Circulating miR-328 expression in newly diagnosed AML patients and healthy controls detected by quantitative real-time polymerase chain reaction (qRT-PCR) analysis. a Expression levels of miR-328 in newly diagnosed AML patients (median expression value 22.99, range: 3.63-242.0; $n=176$ ) and healthy controls (median expression value 89.17, range: 12.05-397.7; $n=70$ ). Data shown are the Median and range, each symbol represents an individual AML patient and healthy controls, horizontal lines indicate median values and range. Mann-Whitney U-test and associated P value is indicated. b Expression levels of miR-328 in 76 AML patients before (mean value 23.40) and after complete remission (CR) ( mean value 46.61). Each symbol represents an individual AML patient at diagnosis and at achievement of $C R$, paired $t$ test and associated $P$ value is indicated 
and those samples with expression above the mean value were assigned to the high expression group (mean expression value 63.03, $\mathrm{n}=51$ ). As shown in Table 1 , low levels of miR-328 were associated with a higher white blood cell count and BM blast count $(P=0.026$ and $P=0.003$, respectively), and lower hemoglobin and platelet count $(P=0.004$ and $P=0.022$, respectively). However, other clinical characteristics, including age $(P$ $=0.997)$, gender $(P=0.847)$, FAB subtype $(P=0.909)$, WHO classification $(P=0.074)$ and karyotype classification $(P=0.570)$ were not directly related to the low level of miR-328.

\section{Association between miR-328 expression and clinical outcomes of AML patients}

To investigate the prognostic impact of miR-328 low expression in AML, survival analysis was performed in 176 cases. There were no differences in the OS and RFS between two groups $(P=0.137$ and $P=0.339$, data not shown). Among 176 cases, 124 patients received standard induction chemotherapy, The $\mathrm{CR}$ rate after two cycles of chemotherapy was $44.0 \%(55 / 125)$ in the lowexpression group, compared with $41.2 \%(21 / 51)$ in the high-expression group $(P=0.861)$, there was no significant difference between the two groups. Moreover, the OS of 124 AML patients with high miR-328 expression was shorter than those with low expression, but the difference was not statistically significant $(P=0.176)$. However, among those obtained CR, overall survival curves and relapse-free survival curves in high-miR-328 group $(\mathrm{n}=21)$ and low-miR-328 group $(\mathrm{n}=55)$ are shown in Fig. 2. Patients with low mR-328 expression have shown significantly poorer overall survival $(P=0.022$, Fig. $2 \mathrm{a})$ and shorter relapse-free survival $(P=0.008$, Fig. 2 b) than those with high miR-328 expression.

Univariate analyses showed that higher white blood cell count $(P=0.004)$, lower hemoglobin $(P=0.009)$, platelet count $(P=0.017), \mathrm{BM}$ blast count $(P=0.012)$ and miR-328 level $(P=0.009)$ were significantly associated with OS (Table 2), while higher white blood cell count $(P=0.009)$, lower hemoglobin $(P=0.04)$ and lower miR-328 level $(P=0.01)$ were found to be prognostic factors for RFS (Table 2). Furthermore, the multivariate Cox regression analysis revealed that low miR-328 expression was an independent prognostic factors for both OS (Hazard ratio $[\mathrm{HR}]=2.67 ; 95 \%$ confidence interval $[\mathrm{CI}], 1.12-4.73 ; P=0.017)$ and RFS $(\mathrm{HR}=1.914 ; 95 \% \mathrm{CI}$, 1.01-3.27; $P=0.023$ ) of AML patients. Statistical values of the expression of miR-328 and other clinical parameters derived from Cox stepwise proportional hazards model were indicated in Table 2.

\section{Discussion}

Nowadays, it is becoming evident that expression patterns of microRNAs appears to be a common characteristic of hematological malignancies including leukemias, some of them can be a valuable tool for the diagnosis and prognosis of human cancer $[26,8]$. Recently, it has been reported that microRNAs are circulating in serum/ plasma. Additionally, microRNAs, such as miR-134 [19], miR-218 [27, 28], miR150 and miR-324 [29] in human serum or plasma have been shown to have much stronger stability than high molecular weight RNA due to their resistance to RNase digestion [15]. These findings make microRNAs a potentially non-invasive tools for cancer diagnosis using blood samples [15].
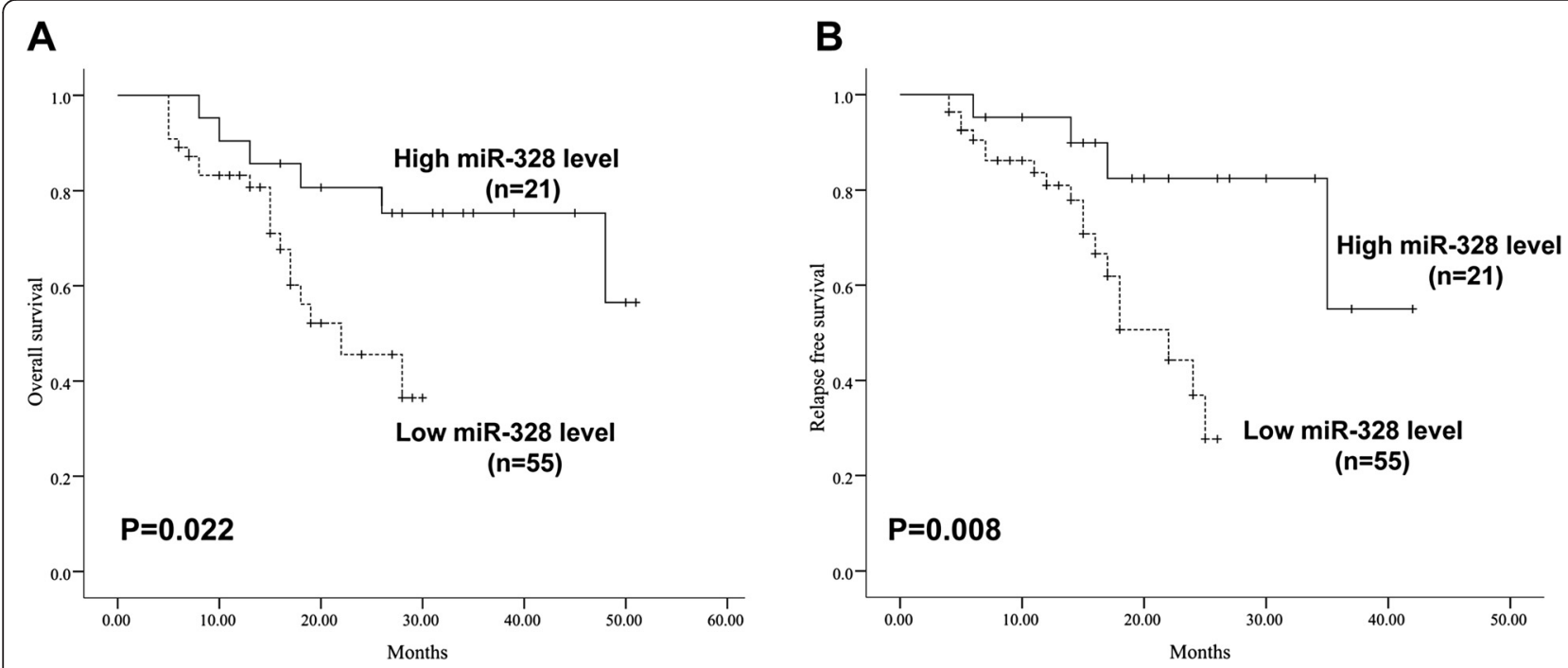

Fig. 2 Kaplan-Meier survival curves for overall survival (OS) and relapse-free survival (RFS) according to circulating miR-328 expression from 76 AML patients in complete remission (CR). a The OS rate of AML patients in CR with high or low miR-328 expression; $\mathbf{b}$ The RFS rate of AML patients in CR with high or low miR-328 expression. The $P$-value was calculated using the log-rank test 
Table 2 Univariate and multivariate analyses of factors associated with survival and relapse of AML patients

\begin{tabular}{|c|c|c|c|c|c|c|c|c|}
\hline \multirow[t]{3}{*}{ Factors } & \multicolumn{4}{|l|}{ OS } & \multicolumn{4}{|l|}{ RFS } \\
\hline & \multirow{2}{*}{$\frac{\text { Univariate }}{P}$} & \multicolumn{3}{|l|}{ Multivariate } & \multirow{2}{*}{$\frac{\text { Univariate }}{P}$} & \multicolumn{3}{|l|}{ Multivariate } \\
\hline & & Hazard Ratio & $95 \% \mathrm{Cl}$ & $P$ & & Hazard Ratio & $95 \% \mathrm{Cl}$ & $P$ \\
\hline Age, years ( $\leq 60$ vs $>60)$ & 0.741 & N.A. & N.A. & N.A. & 0.675 & N.A. & N.A. & N.A. \\
\hline Gender (male vs female) & 0.821 & N.A. & N.A. & N.A. & 0.514 & N.A. & N.A. & N.A. \\
\hline WBC $\left(<10\right.$ vs $\left.\geq 10, \times 10^{9} / \mathrm{L}\right)$ & 0.004 & 1.37 & $0.67-2.04$ & 0.04 & 0.009 & 1.39 & $0.81-2.33$ & 0.039 \\
\hline $\mathrm{HGB}(<80$ vs $\geq 80, \mathrm{~g} / \mathrm{L})$ & 0.009 & 1.25 & $0.66-1.79$ & 0.076 & 0.040 & 1.120 & $0.82-1.63$ & 0.247 \\
\hline $\mathrm{PLT}\left(<50\right.$ vs $\left.\geq 50, \times 10^{9} / \mathrm{L}\right)$ & 0.017 & 1.64 & $1.07-2.87$ & 0.038 & 0.274 & N.A. & N.A. & N.A. \\
\hline Blast in $\mathrm{BM}(<50 \%$ vs $\geq 50 \%)$ & 0.012 & 1.93 & $1.09-3.21$ & 0.041 & 0.213 & N.A. & N.A. & N.A. \\
\hline miR-328 expression (high vs low) & 0.009 & 2.67 & $1.12-4.73$ & 0.017 & 0.01 & 1.914 & $1.01-3.27$ & 0.023 \\
\hline
\end{tabular}

Abbreviations: WBC white blood cell, HGB hemoglobin, $P L T$, platelet

The present study has confirmed, for the first time, that the plasma miR-328 may serve as useful diagnostic and prognostic biomarkers for patients with AML. MiR-328 has been suggested to be a tumor suppressor by targeting proto-oncogene serine/threonine-protein kinase PIM1 and translational regulator protein hnRNP E2 [26]. Eiring et al. reported that miR-328 was down-regulated in chronic myelogenous leukemia blasts, and low expression of miR-328 in CML is associated with progression to the blast crisis phase of the disease [16]. Wu et al. observed that miR-328 expression is decreased in high-grade gliomas and is associated with worse survival in primary glioblastoma [17]. However, miR-328 was also expressed at high levels in several cancers. Ulivi et al. reported that circulating miR-328 expression was significantly higher in non-small cell lung cancer (NSCLC) patients than in healthy donors [18]. Wang et al. found that plasma miR328 concentrations were significantly elevated in acute myocardial infarction (AMI) patients compared to those control subjects [19]. In our research, plasma concentration of miR-328 was markedly downregulated in patients with newly diagnosed AML compared with healthy controls. Moreover, the expression of miR-328 was significantly elevated after chemotherapy when patients achieved $\mathrm{CR}$, suggesting that expression of miR-328 is consistent with tumor burden. Our results were consistent with other studies regarding CML and glioblastoma [17, 16], indicating that miR-328 plays an essential role in the original and/or progression of AML.

MiR-328 is proposed as a suppressor gene because its expression is decreased in several types of cancers and mediates proliferation, invasion and metastasis of cancer cells. It is demonstrated that enforced expression of miR-328 could remarkably attenuate glioma cell proliferation, invasion and migration [30]. MiR-328 could also inhibit epithelialmesenchymal transition (EMT) via targeting CD44 [31]. These findings indicate that miR-328 plays a direct role in the modulation of cancer progression and may be useful as a novel prognostic or progression marker for cancer.
In the current study, we found that downregulatation of miR-328 in AML patients was significantly associated with a higher WBC count and blast count in BM, and lower HGB and PLT counts, which represented more aggressive clinicopathological features. In addition, AML patients with low miR-328 expression tend to have poorer OS and RFS than those with high miR-328 expression, indicating that expression of miR-328 has an important value in AML prognosis classification. In a logistic regression analysis, an association was observed between miR328 expression and the risk of both survival and relapse of AML patients. It was observed that those patients with low expression of miR-328, presented a high risk of OS $(P=0.009)$ and RFS $(P=0.017)$ to AML compared to those patients who had high expression of miR-328 expression. In addition, multivariate analyses performed showed that miR-328 low expression is an independent predictor for OS $(\mathrm{HR}=2.67,95 \% \mathrm{CI}, 1.12-4.73 ; P=0.017)$ and RFS (HR $=1.914,95 \% \mathrm{CI}, 1.01-3.27 ; P=0.023)$ of AML patients, which was in agreement with recent findings in glioblastoma [17]. Taken together, our results suggest that circulating miR-328 maybe function as a suppressor gene in the development of AML, and may have an adverse effect on prognosis in apart of AML patients.

\section{Conclusion}

In summary, our study offers the evidence for the first time that circulating miR-328 is downregualted in AML patients, and lower miR-328 level is closely associated with distinct clinical and biologic characteristics in AML patients. Furthermore, lower miR-328 level is an independent poor prognostic factor for OS and RFS. However, the precise molecular mechanisms by which miR-328 is downregulated in AML need to be further investigation.

\section{Competing interests}

The authors declare that they have no competing interests. 


\section{Authors' contributions}

Conceived and designed the experiments: LL. Performed the experiments: LL, RAC, YPZ, WF. Analyzed the data: LL, RAC. Contributed reagents/ materials/ analysis tools: FX, XQY. Wrote the paper: LL. All authors read and approved the final manuscript.

\section{Acknowledgements}

The authors thank all patients and their families for their participation in this study.

Received: 11 January 2015 Accepted: 9 July 2015

Published online: 17 July 2015

\section{References}

1. Ferrara F, Schiffer CA. Acute myeloid leukaemia in adults. Lancet. 2013;381(9865):484-95. doi:10.1016/S0140-6736(12)61727-9.

2. Meyer SC, Levine RL. Translational implications of somatic genomics in acute myeloid leukaemia. Lancet Oncol. 2014;15(9):e382-94. doi:10.1016/ S1470-2045(14)70008-7.

3. Gregory TK, Wald D, Chen Y, Vermaat JM, Xiong Y, Tse W. Molecular prognostic markers for adult acute myeloid leukemia with normal cytogenetics. J Hematol Oncol. 2009;2:23. doi:10.1186/1756-8722-2-23.

4. Lee RC, Feinbaum RL, Ambros V. The C. elegans heterochronic gene lin-4 encodes small RNAs with antisense complementarity to lin-14. Cell. 1993;75(5):843-54.

5. Bartel DP. MicroRNAs: genomics, biogenesis, mechanism, and function. Cell. 2004;116(2):281-97.

6. Lu J, Getz G, Miska EA, Alvarez-Saavedra E, Lamb J, Peck D, et al. MicroRNA expression profiles classify human cancers. Nature. 2005;435(7043):834-8. doi:10.1038/nature03702.

7. Takada S, Yamashita Y, Berezikov E, Hatanaka H, Fujiwara SI, Kurashina K, et al. MicroRNA expression profiles of human leukemias. Leukemia. 2008:22(6):1274-8. doi:10.1038/sj.leu.2405031.

8. Babashah S, Sadeghizadeh M, Tavirani MR, Farivar S, Soleimani M. Aberrant microRNA expression and its implications in the pathogenesis of leukemias. Cell Oncol (Dordr). 2012;35(5):317-34. doi:10.1007/s13402-012-0095-3.

9. Sun SM, Rockova V, Bullinger L, Dijkstra MK, Dohner H, Lowenberg B, et al. The prognostic relevance of miR-212 expression with survival in cytogenetically and molecularly heterogeneous AML. Leukemia. 2013;27(1):100-6. doi:10.1038/leu.2012.158.

10. Chen XX, Lin J, Qian J, Qian W, Yang J, Ma JC, et al. Dysregulation of miR-124-1 predicts favorable prognosis in acute myeloid leukemia. Clin Biochem. 2014;47(1-2):63-6. doi:10.1016/j.clinbiochem.2013.09.020

11. Li Z, Huang H, Li Y, Jiang X, Chen P, Arnovitz S, et al. Up-regulation of a HOXA-PBX3 homeobox-gene signature following down-regulation of miR-181 is associated with adverse prognosis in patients with cytogenetically abnormal AML. Blood. 2012;119(10):2314-24. doi:10.1182/blood-2011-10-386235.

12. Li Y, Lin J, Yang J, Qian J, Qian W, Yao DM, et al. Overexpressed let-7a-3 is associated with poor outcome in acute myeloid leukemia. Leuk Res. 2013;37(12):1642-7. doi:10.1016/j.leukres.2013.09.022.

13. Qu H, Xu W, Huang $Y$, Yang S. Circulating miRNAs: promising biomarkers of human cancer. Asian Pac J Cancer Prev. 2011;12(5):1117-25.

14. Duttagupta R, Jiang R, Gollub J, Getts RC, Jones KW. Impact of cellular miRNAs on circulating miRNA biomarker signatures. PLoS One. 2011;6(6), e20769. doi:10.1371/journal.pone.0020769.

15. Cheng G. Circulating miRNAs: Roles in cancer diagnosis, prognosis and therapy. Adv Drug Deliv Rev. 2015;81C:75-93. doi:10.1016/ j.addr.2014.09.001

16. Eiring AM, Harb JG, Neviani P, Garton C, Oaks JJ, Spizzo R, et al. miR-328 functions as an RNA decoy to modulate hnRNP E2 regulation of mRNA translation in leukemic blasts. Cell. 2010;140(5):652-65. doi:10.1016/ j.cell.2010.01.007

17. Wu Z, Sun L, Wang H, Yao J, Jiang C, Xu W, et al. MiR-328 expression is decreased in high-grade gliomas and is associated with worse survival in primary glioblastoma. PLoS One. 2012;7(10), e47270. doi:10.1371/ journal.pone.0047270.

18. Ulivi $P$, Foschi $G$, Mengozzi M, Scarpi E, Silvestrini R, Amadori $D$, et al. Peripheral Blood miR-328 Expression as a Potential Biomarker for the Early Diagnosis of NSCLC. Int J Mol Sci. 2013;14(5):10332-42. doi:10.3390/ ijms140510332.
19. He F, Lv P, Zhao X, Wang X, Ma X, Meng W, et al. Predictive value of circulating miR-328 and miR-134 for acute myocardial infarction. Mol Cell Biochem. 2014;394(1-2):137-44. doi:10.1007/s11010-014-2089-0.

20. Swerdllow S, Campo E, Harris NL. WHO classification of tumours of haematopoietic and lymphoid tissues. France: IARC Press. 2008;2008.

21. Bennett JM, Catovsky D, Daniel MT, Flandrin G, Galton DA, Gralnick HR, et al. Proposed revised criteria for the classification of acute myeloid leukemia. A report of the French-American-British Cooperative Group. Ann Intern Med. 1985;103(4):620-5.

22. Lo Coco F, Foa R. Diagnostic and prognostic advances in the immunophenotypic and genetic characterization of acute leukaemia. Eur J Haematol. 1995;55(1):1-9.

23. Slovak ML, Kopecky KJ, Cassileth PA, Harrington DH, Theil KS, Mohamed A, et al. Karyotypic analysis predicts outcome of preremission and postremission therapy in adult acute myeloid leukemia: a Southwest Oncology Group/Eastern Cooperative Oncology Group Study. Blood. 2000:96(13):4075-83

24. Livak KJ, Schmittgen TD. Analysis of relative gene expression data using real-time quantitative PCR and the 2(-Delta Delta C(T)) Method. Methods. 2001;25(4):402-8. doi:10.1006/meth.2001.1262.

25. Schmittgen TD, Livak KJ. Analyzing real-time PCR data by the comparative C(T) method. Nat Protoc. 2008;3(6):1101-8.

26. Adams BD, Kasinski AL, Slack FJ. Aberrant regulation and function of microRNAs in cancer. Curr Biol. 2014;24(16):R762-76. doi:10.1016/ j.cub.2014.06.043.

27. Yu J, Wang Y, Dong R, Huang X, Ding S, Qiu H. Circulating microRNA-218 was reduced in cervical cancer and correlated with tumor invasion. J Cancer Res Clin Oncol. 2012;138(4):671-4. doi:10.1007/s00432-012-1147-9.

28. Xin SY, Feng XS, Zhou LQ, Sun JJ, Gao XL, Yao GL. Reduced expression of circulating microRNA-218 in gastric cancer and correlation with tumor invasion and prognosis. World J Gastroenterol. 2014;20(22):6906-11. doi:10.3748/wjg.v20.i22.6906.

29. Fayyad-Kazan H, Bitar N, Najar M, Lewalle P, Fayyad-Kazan M, Badran R, et al. Circulating miR-150 and miR-342 in plasma are novel potential biomarkers for acute myeloid leukemia. J Transl Med. 2013;11:31. doi:10.1186/1479-5876-11-31

30. Yuan J, Zheng Z, Zheng Y, Lu X, Xu L, Lin L. microRNA-328 is a favorable prognostic marker in human glioma via suppressing invasive and proliferative phenotypes of malignant cells. Int J Neurosci. 2015:1-22. doi:10.3109/00207454.2014.1002610

31. Chen $\mathrm{CH}$, Cheng CY, Chen YC, Sue YM, Liu CT, Cheng TH, et al. MicroRNA-328 inhibits renal tubular cell epithelial-to-mesenchymal transition by targeting the CD44 in pressure-induced renal fibrosis. PLoS One. 2014;9(6), e99802. doi:10.1371/journal.pone.0099802.

\section{Submit your next manuscript to BioMed Central and take full advantage of:}

- Convenient online submission

- Thorough peer review

- No space constraints or color figure charges

- Immediate publication on acceptance

- Inclusion in PubMed, CAS, Scopus and Google Scholar

- Research which is freely available for redistribution 